\title{
Prevalence of resistant hypertension and associated factors for blood pressure control status using Korean ambulatory blood pressure monitoring registry data
}

Sung II Choi', Soon Kil Kim', Sungha Park², Ju Han Kim³, Sang Hyun Ihm², Gwang-il Kim², Woo Shik Kim6, Wook Bum Pyun ${ }^{7}$, Yu-Mi Kim ${ }^{8}$ and Jinho Shin ${ }^{1 *}$

\begin{abstract}
Background: Resistant hypertension $(\mathrm{RH})$ may be one of the cause of the plateau in improving the control rate in hypertension (HT) management. The misdiagnosis of RH by clinic blood pressure (BP) is important clinical problem. Aim of the study were to investigate the prevalence of RH by ambulatory blood pressure monitoring (ABPM) and the factor associated with control status of ambulatory BPs.

Methods: For 1230 subjects taking one or more antihypertensive medication (AHM) enrolled in the Korean Ambulatory Blood Pressure Monitoring (Kor-ABP) registry, the prevalence of RH was calculated which was defined as uncontrolled BP by three AHM classes including diuretic or BP in need of four or more AHM classes. The prevalence determined by clinic versus ambulatory BP was compared.

Results: The age was $59.3 \pm 12.5$ years, and $44.3 \%$ were female $(n=1230)$. Among them 72 subjects were taking three AHM drugs including diuretics and 105 subjects were taking four or more AHM classes. With uncontrolled daytime ambulatory BP in 41 among 72 subjects, prevalence of RH was $11.9 \%$ (146/1230). By using nighttime BP criteria, there was significant difference in the prevalence of RH for clinic versus nighttime BP (146/177 vs. 159/177, $p=0.0124)$. For control status of daytime BP, masked uncontrolled BP was $16.9 \%$ and controlled BP with white-coat effect was $14.1 \%$. For nighttime BP control status, odd ratios for smoking (0.624), drinking (1.512), coronary artery disease (0.604), calcium antagonist (1.705), and loop diuretics (0.454) were all significant.

Conclusion: The prevalence of RH was $11.9 \%$ by daytime BP and it was significantly higher when using nighttime BP criteria. Control status of daytime BP was misclassified in $31.0 \%$. Smoking, drinking, coronary artery disease, calcium antagonist, and loop diuretics were associated with nighttime BP control status.
\end{abstract}

Keywords: Hypertension Resistant to Conventional Therapy, Ambulatory blood pressure monitoring, Masked hypertension, White-coat hypertension, Hypertension

\footnotetext{
* Correspondence: jhs2003@hanyang.ac.kr

${ }^{1}$ Cardiology division, Department of Internal Medicine, Hanyang University

Medical Center, 222 Wangsimni-ro Sungdong-Ku, Seoul \#133-792, South

Korea

Full list of author information is available at the end of the article
} 


\section{Background}

Resistant hypertension ( $\mathrm{RH}$ ) is defined as the hypertension (HT) which cannot be controlled below the target blood pressure even if treated with three or more antihypertensive medications (AHM) classes one of which was diuretics or HT which requires four or more AHM classes regardless whether the blood pressure was controlled or not [1]. In recent years, the trend in the control rate of HT in the treated patient has reach a plateau just below $70 \%$ in Korean population [2]. Because the control of HT is essential component in preventing cardiovascular events, the obstacle against improving the control rate of HT is a very important clinical issue and the epidemiologic study is also very important to provide a basis for setting up management strategy of $\mathrm{RH}$.

As shown in the recent clinical trial, the learning from the misdiagnosis of $\mathrm{RH}$ using only clinic BP made out-ofoffice BP measurement, preferably ABPM, as the essential component of the evaluation before adopting the new therapeutic modality [3]. So far, there are few studies for the prevalence of the $\mathrm{RH}$ by using ABPM in Korean situation. And the amount of the diagnostic error in using clinic BP to diagnose $\mathrm{RH}$ was either unknown in general. And as long as the accurate diagnosis of $\mathrm{RH}$ using ABPM is concerned, there are few reports regarding the demographic or clinical factors related to the RH.

So the aim of the present study is, firstly, to identify the true prevalence of $\mathrm{RH}$ by analyzing the multicenter $\mathrm{ABPM}$ registry data in Korea (Kor- $\mathrm{ABP}$ registry) and to identify the diagnostic errors of clinic BP in the diagnosis of RH. Secondly, it is to identify the demographic and clinical factor related to the treatment resistant condition in the subject treated three or more AHM classes.

\section{Methods}

\section{Subjects}

Among 3766 subjects who were enrolled for Kor-ABP registry during the period from 1st August, 2009 to the end of 2012, 1242 subjects with relevant clinical information and who were treated with at least one AHM were selected for the study. The subject was registered by 27 referral hospitals as described in the previous study [4]. The exclusion criteria was estimated glomerular filtration rate (eGFR) calculated by CKD-EPI equation [5] less than $30 \mathrm{ml} / \mathrm{min} / 1.73 \mathrm{~m} 2$ and finally 1230 subjects was analyzed for the study.

\section{Clinical and laboratory variables}

Information included from the medical records covered: age, gender, height, weight, abdominal circumference, presence of diabetes mellitus (DM), hyperlipidemia, presence of clinical cardiovascular disease, time of diagnosis, mode of treatment, and prescribed medications.
And the questionnaire for each subjects included: smoking status, alcohol intake, extent of physical exercise, family history of HT and premature cardiovascular death, past medical history; HT, DM, hyperlipidemia, stroke, coronary artery disease (CAD), and heart failure.

The collected information for the AHM was the commercial names of the drugs and dosing at the time of enrollment.

\section{Clinic BP and ABPM data}

Clinic BP was measured using an A\&D UA-767, which passed European Society of Hypertension protocol and International Protocol [6]. Clinic BP was defined as the average BP of two measurements one minute apart, with 5 min' rest before the first measurement.

ABPM data were gathered as the form of raw data files uploaded to the study website. The device used in the study institute was all recommended as summarized in the website http://www.dableducational.org or passed the recommended validation protocols [7]. The raw data of ABPM were regarded as valid only when at least 14 readings of the awake blood pressure from 8AM to 9PM were available after omitting erroneous readings according to the following criteria [8]:

1) pulse rate above 120 beats per minute

2) systolic or diastolic blood pressure more than $25 \mathrm{mmHg}$ above or below the previous and subsequent readings

3) pulse pressure below $15 \mathrm{mmHg}$ [9].

\section{Definition of $\mathrm{RH}$ and groups according to the control status of BP}

The definition of the resistant hypertension was uncontrolled BP even if using three drug classes one of which included diuretics or the $\mathrm{BP}$ status requiring treatment using four or more drugs regardless of the control status [1]. Optimal dosage criterion was counted only when the dosage of the each drug was $>50 \%$ of the maximum recommended or approved dose for hypertension [10]. For example, for Angiotensin converting enzyme inhibitor (ACEI), ramipril $5 \mathrm{mg}$ or perindopril $4 \mathrm{mg}$ were minimal dosage needed for optimal treatment. Similarly, for example, losartan $50 \mathrm{mg}$, valsartan $80 \mathrm{mg}$, amlodipine $5 \mathrm{mg}$, diltiazem $90 \mathrm{mg}$, bisoprolol $2.5 \mathrm{mg}$, carvedilol 12.5 $\mathrm{mg}$, nebivolol $2.5 \mathrm{mg}$, dihydrochlorthiazide $12.5 \mathrm{mg}$, furosemide $20 \mathrm{mg}$, torsemide $5 \mathrm{mg}$, indapamide $2.5 \mathrm{mg}$ spironolacton $12.5 \mathrm{mg}$ were the minimal required dosage.

$\mathrm{BP}$ was categorized as controlled and uncontrolled status according to the various criteria. By clinic BP criterion, the BP lowered below 140/90 $\mathrm{mmHg}$ was regarded as controlled status. As for daytime, nighttime, and $24 \mathrm{~h}$ BPs, the BPs lowered below 135/85 mmHg, 120/70 $\mathrm{mmHg}$, and $130 / 80 \mathrm{mmHg}$ were regarded as controlled status, respectively $[2,11]$. 
For the subjects taking three or more AHM including diuretics, according to the control status defined by various criteria, the subject with controlled BP status was grouped as responsive group, whereas the subject with uncontrolled BP status was grouped as the resistant group.

\section{Definitions of the clinical conditions}

Dyslipidemia was defined as the total cholesterol $\geq 240$ $\mathrm{mg} / \mathrm{dL}$, triglyceride $\geq 150 \mathrm{mg} / \mathrm{dL}, \mathrm{HDL}<45 / 50 \mathrm{mg} / \mathrm{dL}$ in male and female, respectively, or the use of statin. The diabetes was defined as fasting blood glucose $\geq 126 \mathrm{mg} /$ $\mathrm{dL}$, hemoglobin $\mathrm{A} 1 \mathrm{C}>6.5 \%$, or the use of antidiabetic medication. The chronic kidney disease (CKD) was defined as eGFR calculated by CKD-EPI equation between 30 and $60 \mathrm{ml} / \mathrm{min} / 1,73 \mathrm{~m} 2$ [5]. Smoking was defined as current smoker and drinking was defined as current drinking by the questionnaire. Regular physical exercise was defined as the three or more times of structured exercise per week. Metabolic syndrome (Mets) was defined when the subject had three or more components of impaired fasting blood glucose or diabetes mellitus, obesity defined by increased abdominal circumference $(\geq 90 \mathrm{~cm}$ in male; $\geq 80 \mathrm{~cm}$ in female) or $\mathrm{BMI} \geq 25 \mathrm{~kg} / \mathrm{m} 2$, triglyceride level $\geq 150 \mathrm{mg} / \mathrm{dL}$, low HDL level, and blood pressure $\geq 130 / 85 \mathrm{mmHg}$ or a history of hypertension [12]. Global cardiovascular risk (GCR) profiles were determined according to the 2013 Korean Society of Hypertension Guideline [2].

The study protocol was approved by the clinical research ethics committees in all hospitals involved in the study.

\section{Statistical analyses}

All data were expressed as mean \pm standard deviation. The statistical significance of the differences in mean values was evaluated using student $t$-test or analysis of variance (ANOVA). The chi square test and Fisher's exact test were used to ascertain the statistical significance of the categorical difference between groups. The differences in diagnostic category between clinic versus ambulatory BPs were evaluated using McNemar's test. Test for inter-rater reliability using the kappa test statistics with $95 \%$ confidence interval were performed to determine the consistency of the two methods of measuring blood pressure.

Multiple logistic regression analysis was performed to examine the association between resistant group defined by various BP criteria and the clinical factors. The independent variable included age, sex, Mets, family history of HTN, physical activity, drinking and smoking status, DM, CKD, history of stroke, CAD, heart failure, the use of ACEI or angiotensin receptor blocker (ARB) as renin angiotensin system (RAS) blockade, beta blocker (BB), calcium channel blocker (CCB), and loop diuretics, and the use of optimal dosage. Statistical significance was defined by a confidence interval of $95 \%$ and $p<0.05$. All data processing and analysis were performed using SAS 9.4 (SAS Institute Inc., Cary, NC, USA).

\section{Results}

General characteristics of the study subjects

The age was $59.3 \pm 12.5$ years, and $44.3 \%$ were female $(n=$ 1230). Body mass index was $25.2 \pm 3.7$ in male and $24.9 \pm$ 3.6 in female $(p=0.0984)$. Smoking and drinking were more frequent in male than female $(11.8 \%$ vs $0.8 \%, p<0.0001$ and $29.5 \%$ vs $7.2 \%, p<0.0001$ ). Family history of HT was present in $46.0 \%$. High GCR was $83.3 \%$ and coronary artery disease, stroke and heart failure were $25.7 \%, 12.6 \%$, and $4.8 \%$, respectively. CKD was observed in $10.6 \%$.

ARBs prescribed were irbesartan (23.1\%), olmesartan (19.0\% ), telmisartan (15.7\%), valsartan (15.7\%), losartan (12.4\%), candesartan (8.3\%), fimasartan (1.7 \%), and eprosartan (4.1\%). Fixed drug combination was prescribed in $48.7 \%$ for ARB and diuretics. The majority of ACEI was perindopril (70.5\%). BBs prescribed were bisoprolol (39.3\%), carvediolol(29.9\%), nebivolol(12.0\%), atenolol(12.0\%), and etc(2.5\%). CCBs prescribed were amlodipine $(49.1 \%)$, slow release form of nifedipine $(15.7 \%)$, diltiazem $(8.3 \%)$, and etc (26.8\%). Diuretics prescribed were dihydrochlorthiazide (65.9\%), loop diuretics $(17.4 \%)$, indapamide $(7.5 \%)$, and spironolactone (6.6\%).

As shown in Table 1, among 1230 subjects, 72 subjects took three drug classes one of which included diuretics and 105 subjects took four or more drug classes. As for clinical BP, BP in 41 among 72 subjects were uncontrolled.

Table 1 The uses of antihypertensive medications in 1230 study subjects according to the optimal disage criteria

\begin{tabular}{|c|c|c|c|}
\hline & $\begin{array}{l}\text { Count by } \\
\text { any dosage }\end{array}$ & $\begin{array}{l}\text { Count by } 50 \% \text { or more } \\
\text { of recommended dose }\end{array}$ & $p^{*}$ \\
\hline $\begin{array}{l}\text { Angiotensin receptor } \\
\text { blocker }\end{array}$ & 714 (57.9 \%) & 705 (57.2 \%) & 0 \\
\hline ACE inhibitor & 166 (13.5 \%) & 157 (12.7 \%) & 0 \\
\hline Beta blocker & 530 (43.0 \%) & 456 (37.0 \%) & $\begin{array}{l}< \\
0.0001\end{array}$ \\
\hline Calcium antagonist & $732(59.4 \%)$ & 727 (59.0 \%) & 0.03 \\
\hline Dihydrochlorthiazide & $259(21.0 \%)$ & 235 (19.1\%) & $<0.0001$ \\
\hline Indapamide & 19 (1.5\%) & 19 (1.5 \%) & 0 \\
\hline Loop diuretics & 49 (4.0 \%) & 47 (3.8 \%) & 0.16 \\
\hline Spironolactone & 20 (1.6 \%) & 20 (1.6 \%) & 0 \\
\hline \multicolumn{4}{|l|}{$\begin{array}{l}\text { Categories of } \\
\text { antihypertensive } \\
\text { drug regimen }\end{array}$} \\
\hline $\begin{array}{l}3 \text { drugs including } \\
\text { diuretics }\end{array}$ & 72 (5.9 \%) & 83 (6.7 \%) & $<0.0001$ \\
\hline 4 drugs & $105(8.5 \%)$ & $83(6.7 \%)$ & \\
\hline $\begin{array}{l}\text { other } 1 \text { or } \\
\text { more drugs }\end{array}$ & 1053(85.6 \%) & 1064(86.5 \%) & \\
\hline
\end{tabular}

ACE angiotensin converting enzyme, * $\mathrm{p}$ value for McNemar chi square test 
Table 2 Agreement between clinic blood pressure and ambulatory blood pressure to classify resistant hypertension

\begin{tabular}{llllll}
\hline & & \multicolumn{2}{c}{ Clinic BP } & $p^{*}$ & $\begin{array}{c}\text { Kappa } \\
\text { (95\% confidence interval) }\end{array}$ \\
\cline { 3 - 5 } Daytime BP & Non-RH & $19(10.7 \%)$ & $12(6.8 \%)$ & 0.53 \\
Nighttime BP & RH & $12(6.8 \%)$ & $134(75.7 \%)$ & 1 & $(0.3658 \sim 0.6956)$ \\
& Non-RH & $11(6.2 \%)$ & $7(4.0 \%)$ & 0.37 & $(0.1813 \sim 0.5539)$ \\
24 h BP & RH & $20(11.3 \%)$ & $139(78.5 \%)$ & 0.01 & 0.53 \\
& Non-RH & $17(9.6 \%)$ & $8(10.2 \%)$ & & $(0.3638 \sim 0.7049)$ \\
\hline
\end{tabular}

$\mathrm{BP}$ blood pressure, $\mathrm{RH}$ resistant hypertension defined by uncontrolled BP by each criteron or BP control needing four or more antihypertensive medications. ${ }^{*}, \mathrm{P}$ for McNemar test

When the dosage of each drug $\geq 50 \%$ of the maximum recommended or approved dose for hypertension was considered effective treatment, 83 subjects took three drugs including diuretics and 83 subjects took four or more drug classes and the categorization was differed (McNemar $p<0.0001$ ). As for clinic BP, BP of 42 among 83 subjects taking three drugs including diuretics were uncontrolled.

\section{Prevalence of RH}

BP in 41 (56.9\%), 41 (56.9\%), 54(75.0\%), and 47(65.2\%) subjects of the 72 subject taking three AHMs including diuretics were not controlled by clinic, daytime, nighttime, and $24 \mathrm{~h} \mathrm{BP}$ criteria (Table 2). Considering 105 subjects taking four or more drugs, among the HT subject taking at least one AHM, the prevalence of RH was $11.9 \%$ (146/ 1230), $11.9 \%$ (146/1230), $12.9 \%$ (159/1230), and $12.3 \%$ $(152 / 1230)$ according to the criteria by clinic, daytime, nighttime, and $24 \mathrm{~h}$ BPs, respectively. But Among the subjects taking three or more AHMs, the prevalence of the RH was $82.4 \%$ (146/177), $82.4 \%$ (146/177), 89.8 \% (159/ $177)$, and $85.9 \%(152 / 177)$ according to the criteria by clinic, daytime, nighttime, and $24 \mathrm{~h}$ BPs, respectively. Because all subjects taking four or more drugs are classified as $\mathrm{RH}$, the prevalence can be sensitive to the control status of the 72 subject taking three drugs including diuretics.

For the subject taking optimal dosage, the prevalence of RH was $10.1 \%$ (125/1230), $10.4 \%$ (129/1230), $11.2 \%$
(139/1230), $10.8 \%(134 / 1230)$ according to the criteria by clinic, daytime, nighttime, and $24 \mathrm{~h}$ BPs, respectively.

\section{Agreement of clinic BP and ambulatory BP for the diagnosis of $\mathrm{RH}$}

According to the definition of $\mathrm{RH}$, the subject with uncontrolled BP among 72 subjects taking three AHMs including diuretics and all of the subjects taking four or more AHMs $(n=105)$ were classified as RH. As shown in Table 3, the diagnosis rate was significantly different when using nighttime BP criterion even though kappa index was similar and poorly agreed.

\section{Agreement of the control status in the subject taking three or more AHMs}

As shown in Table 3, the control status defined by nighttime and $24 \mathrm{~h} \mathrm{BP}$ criteria were significantly different compared to the status defined by clinic BP criterion. At least more than $50 \%$ of the subject taking three or more AHMs showed consistently uncontrolled BP regardless of the kind of the criteria applied. Among 177 subjects taking three or more drugs, 117(66.1\%), 122(68.9\%), 140(79.1\%), and $132(74.5 \%)$ were uncontrolled by clinic, daytime, nighttime, $24 \mathrm{~h}$ BP criteria. The kappa statistics suggested poor agreement showing that 31.0\% $(n=55), 33.4 \%(n=59)$, and $28.8 \%(n=51)$ were misclassified as uncontrolled BP due to white-coat effect or misclassified as controlled BP due to masking by the clinic BP. For control status of

Table 3 Agreement between clinic blood pressure and ambulatory blood pressure in the control status in the subject taking three or more drugs

\begin{tabular}{|c|c|c|c|c|c|}
\hline & & \multicolumn{2}{|c|}{ Clinic BP (mmHg) } & \multirow[t]{2}{*}{$p^{*}$} & \multirow{2}{*}{$\begin{array}{l}\text { Kappa } \\
\text { (95\% confidence interval) }\end{array}$} \\
\hline & & $<140 / 90$ & $>=140 / 90$ & & \\
\hline \multirow[t]{2}{*}{ Daytime BP (mmHg) } & $<135 / 85$ & 30 (16.9\%) & $25(14.1 \%)$ & & 0.29 \\
\hline & $>=135 / 85$ & 30 (16.9\%) & 92 (52.1\%) & 0.5 & $(0.1443 \sim 0.4402)$ \\
\hline \multirow[t]{2}{*}{ Nighttime BP (mmHg) } & $<120 / 70$ & 19 (10.7\%) & 18 (10.2\%) & & 0.18 \\
\hline & $>=120 / 70$ & $41(23.2)$ & 99 (55.9\%) & 0 & $(0.0328 \sim 0.3264)$ \\
\hline \multirow[t]{2}{*}{24 h BP (mmHg) } & $<130 / 80$ & $27(15.3 \%)$ & $18(10.2 \%)$ & & 0.32 \\
\hline & $>=130 / 80$ & $33(18.6 \%)$ & 99 (55.9\%) & 0.04 & $(0.1680 \sim 0.4627)$ \\
\hline
\end{tabular}


Table 4 The comparison according to control status by daytime blood pressure of $135 / 85 \mathrm{mmHg}$ or greater in response to three or more antihypertensive medications including diuretics

\begin{tabular}{|c|c|c|c|}
\hline & $\begin{array}{l}\text { Controlled } \\
\text { BP group }\end{array}$ & $\begin{array}{l}\text { Uncontrolled } \\
\text { BP group }\end{array}$ & $p$ \\
\hline & $(N=55)$ & $(N=122)$ & \\
\hline Age (yr) & $61.6 \pm 11.2$ & $61.2 \pm 10.4$ & 0.84 \\
\hline Body mass index (Kg/m2) & $25.7 \pm 4.1$ & $25.6 \pm 3.3$ & 0.83 \\
\hline Abdominal circumference $(\mathrm{cm})$ & $93.8 \pm 10.9$ & $91.9 \pm 9.3$ & 0.28 \\
\hline Clinic systolic BP (mmHg) & $135.1 \pm 18.2$ & $153.8 \pm 24.1$ & $<0.0001$ \\
\hline Clinic diastolic BP (mmHg) & $80.2 \pm 12.3$ & $91.5 \pm 16.1$ & $<0.0001$ \\
\hline Heart rate (beats per minute) & $73.7 \pm 14.4$ & $75.2 \pm 14.8$ & 0.58 \\
\hline Potassium (mEq/L) & $4 \pm 0.4$ & $4.1 \pm 0.5$ & 0.37 \\
\hline Creatinine (mg/dL) & $1 \pm 0.3$ & $0.9 \pm 0.2$ & 0.14 \\
\hline Cholesterol (mg/dL) & $171.4 \pm 40.7$ & $182.9 \pm 44.9$ & 0.17 \\
\hline Fasting blood glucose (mg/dL) & $114.2 \pm 34$ & $109.6 \pm 34.6$ & 0.53 \\
\hline Hemoglobin A1C (\%) & $6.6 \pm 1.9$ & $6.3 \pm 1.1$ & 0.37 \\
\hline Triglyceride (mg/dL) & $128.3 \pm 54.2$ & $148.4 \pm 81$ & 0.19 \\
\hline High density lipoprotein (mg/dL) & $46.7 \pm 13.1$ & $45.8 \pm 10$ & 0.67 \\
\hline Daytime systolic BP (mmHg) & $121.8 \pm 7.9$ & $149.6 \pm 15.2$ & $<0.0001$ \\
\hline Daytime diastolic BP (mmHg) & $74.8 \pm 5.3$ & $90.9 \pm 11.7$ & $<0.0001$ \\
\hline Daytime heart rate (bpm) & $71.4 \pm 9.6$ & $73.9 \pm 10.2$ & 0.14 \\
\hline Nighttime systolic BP (mmHg) & $115.5 \pm 14$ & $138.5 \pm 20.5$ & $<0.0001$ \\
\hline Nighttime diastolic BP (mmHg) & $71 \pm 9.7$ & $83.6 \pm 15.4$ & $<0.0001$ \\
\hline Nighttime heat rate (bpm) & $61.8 \pm 10.3$ & $64.8 \pm 11.3$ & 0.11 \\
\hline 24 h systolic BP (mmHg) & $121 \pm 8.9$ & $146.9 \pm 15.3$ & $<0.0001$ \\
\hline 24 h diastolic BP (mmHg) & $73.9 \pm 5.8$ & $89.5 \pm 12.7$ & $<0.0001$ \\
\hline $24 \mathrm{~h}$ heart rate (bpm) & $68.6 \pm 9.3$ & $71.3 \pm 10$ & 0.11 \\
\hline Smoking & $14.6 \%$ & $11.5 \%$ & 0.57 \\
\hline Drinking & $29.1 \%$ & $32.8 \%$ & 0.62 \\
\hline Regular physical activity & $43.6 \%$ & $36.9 \%$ & 0.39 \\
\hline Family history of SCD & $3.6 \%$ & $6.6 \%$ & 0.35 \\
\hline Family history of hypertension & $36.4 \%$ & $46.7 \%$ & 0.2 \\
\hline Metabolic syndrome & $47.3 \%$ & $44.3 \%$ & 0.71 \\
\hline High global CV risk & $89.1 \%$ & $84.3 \%$ & 0.4 \\
\hline Cardiovascular disease history & $60.0 \%$ & $45.1 \%$ & 0.07 \\
\hline Coronary artery disease & $36.4 \%$ & $26.5 \%$ & 0.18 \\
\hline Stroke & $14.8 \%$ & $9.0 \%$ & 0.25 \\
\hline Heart failure & $18.5 \%$ & $5.8 \%$ & 0.01 \\
\hline \multicolumn{4}{|l|}{ Antihypertensive medication } \\
\hline ACE inhibitor & $14.5 \%$ & $22.1 \%$ & 0.24 \\
\hline Angiotensin receptor blocker & $69.1 \%$ & $78.7 \%$ & 0.17 \\
\hline Beta blocker & $56.4 \%$ & $74.6 \%$ & 0.02 \\
\hline Calcium antagonist & $50.9 \%$ & $69.7 \%$ & 0.02 \\
\hline Diuretics & $100.0 \%$ & $99.2 \%$ & 0.5 \\
\hline Thiazide & $70.9 \%$ & $78.5 \%$ & 0.27 \\
\hline
\end{tabular}

Table 4 The comparison according to control status by daytime blood pressure of $135 / 85 \mathrm{mmHg}$ or greater in response to three or more antihypertensive medications including diuretics (Continued)

\begin{tabular}{lrrc}
\hline Indapamide & $7.3 \%$ & $5.8 \%$ & 0.71 \\
Spironolactone & $5.5 \%$ & $6.6 \%$ & 0.77 \\
Loop diuretics & $21.8 \%$ & $10.7 \%$ & 0.05 \\
Alpha blocker & $1.8 \%$ & $2.5 \%$ & 0.41 \\
Vasodilator & $0.0 \%$ & $2.5 \%$ & 0.55 \\
Aspirin & $50.1 \%$ & $54.1 \%$ & 0.76 \\
Statin & $40.0 \%$ & $40.9 \%$ & 0.9 \\
ACE angitensin converting enzyme, BP blood pressure, $C V$ cardiovascular, SCD \\
sudden cardiac death
\end{tabular}

daytime BP, masked uncontrolled BP was $16.9 \%$ and controlled BP with white-coat effect was $14.1 \%$. Masked uncontrolled HT was more frequently observed when using nighttime BP criterion than daytime BP criterion (16.9\% vs. $23.1 \%, p=0.0343$ ). Because daytime BPs in 31 subjects were controlled as shown in Table 2, among 105 subjects taking four or more drugs, 91 subjects $(86.6 \%)$ as shown in Table 3 were uncontrolled in daytime BP.

\section{Characteristics of the controlled versus uncontrolled BP groups}

As shown in Table 4, when the criteria for control status by daytime ambulatory BP was applied, there was no significant difference between controlled BP group and uncontrolled BP group in terms of demographic and clinical profiles except for BP levels. Significantly higher $\mathrm{CAD}$ and heart failure were observed in controlled $\mathrm{BP}$ group than uncontrolled BP group. In terms of AHMs, in uncontrolled $\mathrm{BP}$ group, $\mathrm{BB}$ and $\mathrm{CCB}$ were more frequently prescribed whereas loop diuretics were more frequently prescribed in controlled BP group.

\section{The factors related to control status}

As shown in Table 5 and Fig. 1, smoking was related to controlled BP group for nighttime BP whereas drinking was related to the uncontrolled BP group for nighttime and $24 \mathrm{~h}$ BPs. History of CAD is associated with controlled BP group consistently regardless of the criteria applied. Heart failure was related to controlled BP group for daytime BP. Regarding the class of AHM, CCB was consistently related to the uncontrolled $\mathrm{BP}$ group regardless of the criteria applied. Specifically for nighttime $\mathrm{BP}, \mathrm{CCB}$ was the only AHM class related to uncontrolled $\mathrm{BP}$ group. ACEI or ARB was also related to uncontrolled BP group by any criteria except for the nighttime BP. Interestingly, use of loop diuretics was associated with controlled BP group for nighttime BP. 
Table 5 Multiple logistic regression analysis and odds ratios for the factors associated with the resistant group to antihypertensive theraphy using 3 or more antihypertensive medications including diuretics

\begin{tabular}{lllll}
\hline & Clinic BP & Daytime BP & Nighttime BP & $24 \mathrm{~h} \mathrm{BP}$ \\
& ${ }^{3} 140 / 90 \mathrm{mmHg}$ & $3.135 / 85 \mathrm{mmHg}$ & $3120 / 70 \mathrm{mmHg}$ & ${ }^{3} 130 / 80 \mathrm{mmHg}$ \\
\hline Age & $0.989[0.978 \sim 1.001]$ & $0.992[0.98 \sim 1.004]$ & $0.996[0.983 \sim 1.009]$ & $0.992[0.98 \sim 1.005]$ \\
Male sex & $0.956[0.71 \sim 1.288]$ & $1.221[0.901 \sim 1.655]$ & $1.171[0.836 \sim 1.639]$ & $1.157[0.84 \sim 1.594]$ \\
Metabolic syndrome & $0.965[0.743 \sim 1.253]$ & $1.037[0.793 \sim 1.357]$ & $0.963[0.717 \sim 1.294]$ & $1.043[0.786 \sim 1.385]$ \\
Smoking & $1.202[0.791 \sim 1.827]$ & $1.161[0.75 \sim 1.797]$ & $0.624[0.399 \sim 0.976]$ & $1.008[0.639 \sim 1.59]$ \\
Drinking & $1.135[0.843 \sim 1.528]$ & $1.242[0.913 \sim 1.688]$ & $1.512[1.072 \sim 2.133]$ & $1.4[1.01 \sim 1.942]$ \\
Regular physical exercise & $0.826[0.636 \sim 1.073]$ & $0.855[0.654 \sim 1.119]$ & $0.99[0.735 \sim 1.335]$ & $0.869[0.654 \sim 1.153]$ \\
Family history of hypertension & $1.231[0.947 \sim 1.6]$ & $1.131[0.865 \sim 1.48]$ & $1.121[0.834 \sim 1.507]$ & $1.169[0.88 \sim 1.552]$ \\
Diabetes mellitus & $0.917[0.685 \sim 1.226]$ & $0.791[0.589 \sim 1.062]$ & $1.172[0.837 \sim 1.64]$ & $0.919[0.673 \sim 1.256]$ \\
History of stroke & $1.166[0.786 \sim 1.73]$ & $1.042[0.701 \sim 1.549]$ & $1.391[0.874 \sim 2.214]$ & $1.063[0.699 \sim 1.617]$ \\
History of coronary artery disease & $0.619[0.458 \sim 0.835]$ & $0.632[0.465 \sim 0.859]$ & $0.604[0.431 \sim 0.846]$ & $0.605[0.44 \sim 0.832]$ \\
History of heart failure & $0.896[0.495 \sim 1.623]$ & $0.559[0.309 \sim 1.009]$ & $0.638[0.345 \sim 1.179]$ & $0.646[0.354 \sim 1.178]$ \\
CKD & $1.08[0.709 \sim 1.646]$ & $1.025[0.67 \sim 1.569]$ & $1.298[0.79 \sim 2.133]$ & $1.293[0.815 \sim 2.051]$ \\
Beta blocker & $0.962[0.731 \sim 1.265]$ & $0.838[0.634 \sim 1.107]$ & $1.017[0.746 \sim 1.385]$ & $0.742[0.554 \sim 0.994]$ \\
Calcium antagonist & $1.527[1.176 \sim 1.982]$ & $1.589[1.218 \sim 2.074]$ & $1.705[1.271 \sim 2.288]$ & $1.411[1.066 \sim 1.868]$ \\
ACE inhibitor or ARB & $1.42[1.083 \sim 1.863]$ & $1.479[1.122 \sim 1.95]$ & $1.273[0.938 \sim 1.728]$ & $1.376[1.03 \sim 1.837]$ \\
Loop diuretics & $0.755[0.405 \sim 1.408]$ & $0.54[0.289 \sim 1.011]$ & $0.454[0.24 \sim 0.861]$ & $0.541[0.288 \sim 1.014]$ \\
Optimal dosage use & $1.283[0.838 \sim 1.965]$ & $1.285[0.836 \sim 1.976]$ & $1.15[0.72 \sim 1.838]$ & $1.086[0.696 \sim 1.696]$ \\
\hline ACE ango & &
\end{tabular}

$A C E$ angiotensin converting enzyme, $A R B$ angiotensin receptor blocker, $B P$ blood pressure, CKD chronic kidney disease with eGFR between 30 and $60 \mathrm{ml} / \mathrm{min} / 1.73 \mathrm{~m} 2$

\section{Discussion}

The most important finding of the present study is that the prevalence of $\mathrm{RH}$ is $11.9 \%$, which was within the $95 \%$ confidence interval of the prevalence of the previous reports [13]. As noted in that meta-analysis, as a cause of the pseudo- $\mathrm{RH}$, the role of ABPM to avoid misdiagnosis was clearly demonstrated in our study [13]. As shown in our study (Table 3), the misclassification for the control status is expected in about $30 \%$ by the presence of whitecoat effect or masked uncontrolled hypertensions without using ABPM or other out-of-office BP measurement, possibly, home BP monitoring. As for the role of ABPM in $\mathrm{RH}$, the misdiagnosis of $\mathrm{RH}$ was relatively small $(6.8 \%)$ each for white-coat effect and masked uncontrolled BP because all of the subject taking four or more drugs were classified as RH regardless of the control status. So the role of ABPM is more important when evaluating the control status which is closely related to the choice of the further therapy. Moreover once the BP was not controlled by one or two AHMs, our study showed that the response to the treatment by using three or more AHMs even including diuretics in almost all subjects was quite poor (control rate less than $31 \%$ ). Much more effective treatment modality, such as more active use of aldosterone antagonist and/or renal denervation for selected patients should be prescribed accurately for the right patient using ABPM $[14,15]$.
And without considering the optimal dosage criteria, the prevalence was overestimated by $1 \sim 2 \%$ in our study which was partially consistent but the difference was not as striking as reported in the previous study [10].

In addition, our study identified the related clinical factor for the uncontrolled BP group in spite of medical therapy using 3 or more AHMs including diuretics in a multivariate model including optimal dosage criterion. There was no relation suggested by Mets to uncontrolled BP group. It can be explained by the risk profile of subjects requiring three or more drugs and coronary artery disease patients too different to be compared with the most populations where Mets implied cardiovascular risk [16]. Smoking was usually known to be associated with elevation of daytime BP or masked HT. So smoking could induce overestimation of the daytime BP so that the nighttime BP should be lower than nonsmoker [17]. The relation of smoking to $\mathrm{RH}$ by clinic BP as shown in a previous study was not reproduced in this study probably due to small sample size [18]. The adverse effect of drinking was significant for uncontrolled BP group for nighttime and $24 \mathrm{~h}$ BPs. Because heavy drinking itself can be associated with $\mathrm{RH}$ and because nighttime BP is of prognostic importance [19], further study is needed regarding the amount of alcohol intake and the influence on the nighttime BP in RH [20]. The definition of uncontrolled BP group separately from $\mathrm{RH}$ seems to be 

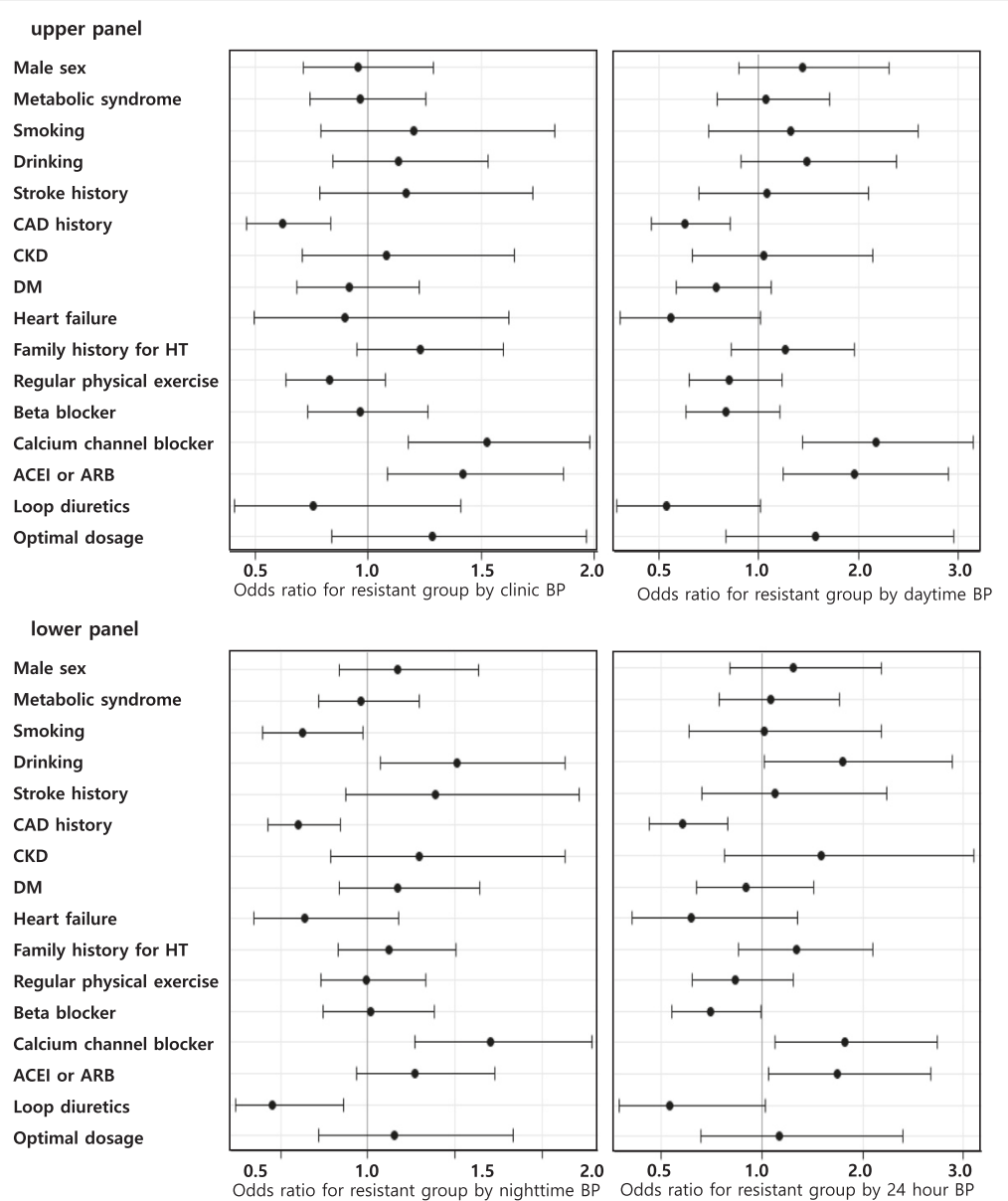

Fig. 1 Demographic and clinical factors related to uncontrolled BP group when treated with three or more antihypertensive medications including diuretics. In the upper panel, CAD history, the use of calcium channel blocker or the use of ACEI/ARB were significant factor for uncontrolled BP group. In the lower left panel, additionally smoking, drinking, and loop diuretics were significantly related factor for uncontrolled BP group defined by nighttime $\mathrm{BP} \geq 120 / 70 \mathrm{mmHg}$. In the lower right panel, CAD history, the use of calcium channel blocker or the use of ACEI/ARB were associated with uncontrolled $\mathrm{BP}$ group defined by $24 \mathrm{~h} \mathrm{BP} \geq 130 / 80 \mathrm{mmHg}$. ACEl, angiotensin converting enzyme inhibitor; ARB, angiotensin receptor blocker; BP, blood pressure ; CAD, coronary artery disease; CKD, chronic kidney disease; DM, diabetes mellitus; $H T$, hypertension

better to avoid confusion but because only a minor proportion of BP in the subject treated with four or more drugs was controlled, its clinical implication needs further study.

Our study showed that CAD is consistently associated with controlled group. This finding can be potentially explained by routine use of beta blockade or RAS blockade when BP level is tolerable. Another interesting finding of our study is that heart failure is associated with daytime controlled BP group. This finding also can be explained by the standard regimen of heart failure which includes RAS blockade, beta blocker, and diuretics which mimics RH situation. Specifically, systolic heart failure patients are so sensitive to the dosage of AHM that they usually have relatively low or well controlled BP. This postulation is partly consistent with the finding that loop diuretics were more frequently prescribed in the controlled BP group. But in RH situation, heart failure itself can be aggravated by high or uncontrolled BP so that multiple drug treatment might not be the result of the standard therapy of heart failure but more likely to be the attempt of controlling $\mathrm{BP}$ as the primary reason for heart failure.

In our study, as an independent variable in the multiple logistic regression analysis, "optimal dosage" was included so that the effect of the suboptimal dosage possibly more frequently used in heart failure or CAD could be adjusted. Because the proportion of the heart failure was $4.8 \%$ in our study, further study is needed for the relationship between heart failure and the $\mathrm{RH}$.

The relation of AHM class to the uncontrolled BP group is very difficult to explain because our study is a cross-sectional study. The association of ARB is different from the $\mathrm{CCB}$ in regard to the nighttime BP. Because $\mathrm{CCB}$ is relatively potent drug to reduce $\mathrm{BP}$, the association might demonstrate the perceived role of $\mathrm{CCB}$ in 
$\mathrm{RH}$. But it also could be the result of secondary failure of BP control from sympathetic activation by using CCB. Further prospective study is needed to answer if this finding is due to the pharmacological property of those drugs such as the half-life or specific effect on the nighttime BP. For the loop diuretics, it is very well known that enough diuretic therapy is very important to deal with RH. Our finding is consistent with the role of diuretics in $\mathrm{RH}$. But because its use in heart failure may be a confounding factor, further study is needed.

In terms of $\mathrm{ABPM}$ versus home BP monitoring issues, our study showed that the nighttime BP criteria can be characterized by increased prevalence of $\mathrm{RH}$ and more clinical factors were related. Even though the diagnostic thresholds by ABPM based on the cardiovascular outcomes have been reported in many studies [21-25], it is reported that the nighttime $\mathrm{BP}$ level has the better predictive power for CV events [26]. So the implications of the RH diagnosed by nighttime BP criteria should be studied in future study for the cardiovascular prognosis.

The prevalence of the masked uncontrolled HT and white-coat controlled HT or treated normalized HT in RH was quite similar to the prevalence in general hypertensive subjects [11,27]. But as shown in our study, because most of the subject had high GCR profile, the clinical implication of masked uncontrolled HTN seems to be much more important to prevent cardiovascular events.

\section{Study limitations}

The present study has several limitations. The generalizability can be limited because Kor-ABP registry data was driven by the tertiary referral center. But considering the RH is generally handled by the referral center, our data can be applied for referral center. And for all negative findings in this study such as the relation of MetS, CKD, or the relation of optimal dosage to the control status, it needs careful interpretation because the sample size seemed to be too small to exclude those associations. Secondly, in RH patient, it is crucial to make a differential diagnosis for secondary HT. But in our study, no standardized approach or reliable exclusion for secondary HT was performed. Thirdly, the information about the adherence as the behavioral factor which is important cause of RH was not available in the present study. Finally the relation of the clinical factors to the treatment resistance is not based on the causality because this study is a cross-sectional study.

\section{Conclusions}

In conclusion, the prevalence of RH was $11.9 \%$ by daytime $\mathrm{BP}$ and it was significantly higher when using nighttime BP criteria. Control status of daytime BP was misclassified in $31 \%$. Smoking, drinking, coronary artery disease, calcium antagonist, and loop diuretics were associated with nighttime BP control status.
Competing interests

The authors declare that they have no competing interests.

\section{Authors' contributions}

SIC, prepare manuscript; SKK, data collection, study hypothesis; SP, data collection, review of manuscript; JHK, data collection, manuscript review; SHI, data collection, manuscript review; GK; data collection, review of manuscript and discussion points; WSK, data collection, review of the data analysis; WBP, data collection, prepare manuscript in part; Y-MK, statistical consultation, study design; JS, study design, data analysis, and hypothesis generation. All authors read and approved the final manuscript.

\section{Acknowledgements}

This study was supported and organized by the Korean Society of Hypertension and the Working group of blood pressure monitoring in Korean Society of Hypertension.

\section{Principal Investigator}

Soon Kil Kim

\section{Investigators}

Sang Hong Baek, Seoul St. Mary's Hospital, The Catholic University of Korea; Sang Hyun Ihm, Y. S. Choi, Byung-Ryul Cho, Kangwon National University Hospital; Woo-Shik Kim, II Suk Sohn, Kyung Hee University School of Medicine; Jin-Won Jeong, Eun Mi Lee, Wonkwang University College of Medicine; J. H. Cho, Soonchun St. Carollo Hospital; Wan Joo Shim, Korea University Medical College Anam Hospital; Young-Kwon Kim, Dongguk University Ilsan Hospital; J. O. Jeong, Chungnam University College of Medicine; Sung Ha Park, Se-Joong Rim, Yonsei Univeresity College of Medicine; Soon Kil Kim, Jinho Shin, Hanyang University College of Medicine; J. H. Jeong, Chosun University College of Medicine; Myeong-Chan Cho, Chungbuk National Univereisty Hospital; Ju Han Kim, Gye Hoon Kim, Chonnam National University Hospital; Gwang-il I Kim, Seoul National University College of Medicine; Dae-Gyun Park, Kangdong Sacred Hospital, Hallym University; Sahng Lee, Eulji University College of Medicine; Wook Bum Pyun, Ewha Womans University School of Medicine; W. Kim, Gwangjoo Veterans Hospital; S. J. Yoon, NHIC Ilsan Hospital; S. K. Choi, Inje University College of Medicine; W. H. Kim, Chonbuk National University College of Medicine; J. Y. Kim, Wonju College of Medicine, Yonsei University.

\section{Author details}

${ }^{1}$ Cardiology division, Department of Internal Medicine, Hanyang University Medical Center, 222 Wangsimni-ro Sungdong-Ku, Seoul \#133-792, South Korea. ${ }^{2}$ Department of Internal Medicine, Yonsei University, School of Medicine, Seoul, Korea. ${ }^{3}$ Department of Internal Medicine, Chonnam University, School of Medicine, GwangJu, Korea. ${ }^{4}$ Department of Internal Medicine, Catholic University, College of Medicine, Bucheon, Korea. ${ }^{5}$ Department of Internal Medicine, Seoul National University, School of Medicine, Bundang, Korea. ${ }^{6}$ Department of Internal Medicine, Kyung Hee University, School of Medicine, Seoul, Korea. ${ }^{7}$ Department of Internal Medicine, Ewha Womans University, School of Medicine, Seoul, Korea. ${ }^{8}$ Department of Preventive Medicine, Dong-A University College of Medicine, Busan, Korea.

Received: 10 November 2015 Accepted: 21 January 2016

Published online: 27 January 2016

\section{References}

1. Calhoun DA, Jones D, Textor S, Goff DC, Murphy TP, Toto RD, et al. Resistant hypertension: diagnosis, evaluation, and treatment: a scientific statement from the American Heart Association Professional Education Committee of the Council for High Blood Pressure Research. Circulation. 2008;117:e510-26.

2. Shin J, Park JB, Kim KI, Kim JH, Yang DH, Pyun WB, et al. 2013 Korean Society of Hypertension guidelines for the management of hypertension: part I-epidemiology and diagnosis of hypertension. Clin Hypertens. 2015;21:11.

3. Bakris GL, Townsend RR, Liu M, Cohen SA, D'Agostino R, Flack JM, et al. Impact of renal denervation on 24-hour ambulatory blood pressure: results from SYMPLICITY HTN-3. J Am Coll Cardiol. 2014;64:1071-8.

4. Shin J, Park SH, Kim JH, Ihm SH, Kim Kl, Kim WS, et al. Discordance between ambulatory versus clinic blood pressure according to global cardiovascular risk group. Korean J Intern Med. 2015;30:610-9. 
5. Levey AS, Stevens LA, Schmid CH, Zhang YL, Castro 3rd AF, Feldman HI, et al. A new equation to estimate glomerular filtration rate. Ann Intern Med. 2009;150:604-12.

6. Rogoza AN, Pavlova TS, Sergeeva MV. Validation of A\&D UA-767 device for the self-measurement of blood pressure. Blood Press Monit. 2000;5:227-31.

7. Haensel A, Utech K, Langewitz W. Validation of TONOPORT V blood-pressure measuring monitor in adults. J Hum Hypertens. 2005;19:745-9.

8. Verdecchia P, Schillaci G, Boldrini F, Zampi I, Porcellati C. Variability between current definitions of 'normal' ambulatory blood pressure. Implications in the assessment of white coat hypertension. Hypertension. 1992;20:555-62.

9. Verdecchia P, Angeli F, Cavallini C. Ambulatory blood pressure for cardiovascular risk stratification. Circulation. 2007;115:2091-3.

10. Egan BM, Zhao Y, Li J, Brzezinski WA, Todoran TM, Brook RD, et al. Prevalence of optimal treatment regimens in patients with apparent treatment-resistant hypertension based on office blood pressure in a community-based practice network. Hypertension. 2013;62:691-7.

11. Franklin SS, Thijs L, Hansen TW, Li Y, Boggia J, Kikuya M, et al. Significance of white-coat hypertension in older persons with isolated systolic hypertension: a meta-analysis using the International Database on Ambulatory Blood Pressure Monitoring in Relation to Cardiovascular Outcomes population. Hypertension. 2012;59:564-71.

12. Park SH, Yoon JS, Won KC, Lee HW. Usefulness of glycated hemoglobin as diagnostic criteria for metabolic syndrome. J Korean Med Sci. 2012;27:1057-61.

13. Achelrod D, Wenzel U, Frey S. Systematic review and meta-analysis of the prevalence of resistant hypertension in treated hypertensive populations. Am J Hypertens. 2015;28:355-61.

14. Williams B, MacDonald TM, Morant S, Webb DJ, Sever P, Mclnnes G, et al. Spironolactone versus placebo, bisoprolol, and doxazosin to determine the optimal treatment for drug-resistant hypertension (PATHWAY-2): a randomised, double-blind, crossover trial. Lancet. 2015;386:2059-68.

15. Iliescu R, Lohmeier TE, Tudorancea I, Laffin L, Bakris GL. Renal denervation for the treatment of resistant hypertension: review and clinical perspective. Am J Physiol Renal Physiol. 2015;309:F583-94.

16. Yoon HJ, Ahn Y, Kim KH, Park JC, Choi DJ, Han S, et al. The prognostic implication of metabolic syndrome in patients with heart failure. Korean Circ J. 2013;43:6

17. Hwang E, Choi K, Kang D, Nam G, Jang J, Jeong Y, et al. Prevalence, predictive factor, and clinical significance of white-coat hypertension and masked hypertension in Korean hypertensive patients. Korean J Intern Med. 2007;22:7.

18. Shimbo D, Levitan EB, Booth 3rd JN, Calhoun DA, Judd SE, Lackland DT, et al. The contributions of unhealthy lifestyle factors to apparent resistant hypertension: findings from the Reasons for Geographic And Racial Differences in Stroke (REGARDS) study. J Hypertens. 2013;31:370-6.

19. Fan HQ, Li Y, Thijs L, Hansen TW, Boggia J, Kikuya M, et al. Prognostic value of isolated nocturnal hypertension on ambulatory measurement in 8711 individuals from 10 populations. J Hypertens. 2010;28:2036-45.

20. Sarafidis PA, Bakris GL. Resistant hypertension: an overview of evaluation and treatment. J Am Coll Cardiol. 2008;52:1749-57.

21. Boggia J, Li Y, Thijs L, Hansen TW, Kikuya M, Bjorklund-Bodegard K, et al. Prognostic accuracy of day versus night ambulatory blood pressure: a cohort study. Lancet. 2007;370:1219-29.

22. Dawes MG, Coats AJ, Juszczak E. Daytime ambulatory systolic blood pressure is more effective at predicting mortality than clinic blood pressure. Blood Press Monit. 2006;11:111-8.

23. Mesquita-Bastos J, Bertoquini S, Polonia J. Cardiovascular prognostic value of ambulatory blood pressure monitoring in a Portuguese hypertensive population followed up for 8.2 years. Blood Press Monit. 2010;15:240-6.

24. Kikuya M, Hansen TW, Thijs L, Bjorklund-Bodegard K, Kuznetsova T, Ohkubo $T$, et al. Diagnostic thresholds for ambulatory blood pressure monitoring based on 10-year cardiovascular risk. Circulation. 2007;115:2145-52.

25. Thijs L, Hansen TW, Kikuya M, Bjorklund-Bodegard K, Li Y, Dolan E, et al. The International Database of Ambulatory Blood Pressure in relation to Cardiovascular Outcome (IDACO): protocol and research perspectives. Blood Press Monit. 2007;12:255-62.

26. Dolan E, Stanton A, Thijs L, Hinedi K, Atkins N, McClory S, et al. Superiority of ambulatory over clinic blood pressure measurement in predicting mortality: the Dublin outcome study. Hypertension. 2005;46:156-61.

27. Celis H, Fagard RH. White-coat hypertension: a clinical review. Eur J Intern Med. 2004;15:348-57.

\section{Submit your next manuscript to BioMed Central and we will help you at every step:}

- We accept pre-submission inquiries

- Our selector tool helps you to find the most relevant journal

- We provide round the clock customer support

- Convenient online submission

- Thorough peer review

- Inclusion in PubMed and all major indexing services

- Maximum visibility for your research

Submit your manuscript at www.biomedcentral.com/submit
Biomed Central 